New Doctorial Cancer Research

\title{
Molecular Cytogenetic Analysis of Archival and Fresh Solid Tumor Samples, 82-8072-229-7
}

\author{
Petter Brandal \\ Department of Cancer Genetics, Rikshospitalet-Radiumhospitalet \\ Medical Center, Montebello, Oslo, Norway
}

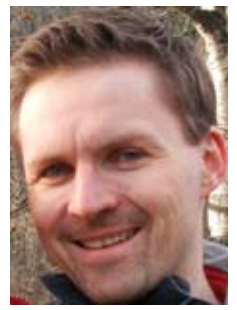

Petter Brandal

Ph.D. Dissertation date: June 16, 2006 Supervisor: Sverre Heim

The acquisition of genetic aberrations by somatic cells is crucially important for neoplastic transformation, and clonal chromosomal aberrations have been detected in most neoplastic entities studied. These nonrandom chromosome aberrations are important pathogenetic information carriers both regarding how neoplasms originate and how they evolve clonally, and they are also gaining importance as diagnostic and prognostic markers. Unfortunately, cells from all tumors, in particular low-incident tumor entities where cytogenetics is not an integrated part of the diagnostic procedure, are not always cultured and the tumor karyotype can therefore not be established. Furthermore, cells from solid tumors are often difficult to grow in vitro, and when successfully cultured, they frequently exhibit very complex karyotypes. It is therefore sometimes of interest to use complementary methods to traditional banding techniques to obtain pathogenetic information about the neoplastic disease process.

In the work leading up to this thesis, the genomes of cells from six different tumor entities were studied. We showed that formalin-fixed, paraffin-embedded archival material may be used for molecular cytogenetic analyses, but that the quality of the archival material is crucially important for eventual success. New karyotypic abnormalities were reported for five of the tumor entities studied and we presented the first-ever karyotyped reninoma, a benign mesenchymal kidney neoplasm, with the following karyotype: 57 64,XX,-X,-1,-4,-6,-9,+10,-11,-13,14,-15,+20,-22[cp10]/60 61,XX,idem,add(19)(p13)[cp2]/46,XX[3] (see Fig. 1). Several lost and gained genomic regions were found in many of the tumors by comparative genomic hybridization, whereas interphase fluorescence in situ hybridization analyses uncovered several numerical chromosome aberrations. In the last study, we analyzed the genomes of four morphological subtypes of lipomatous tumors. Although morphologically different, all of these five tumors had chromosomal aberrations affecting chromosome region 8q11-13 (see Fig. 1), possibly leading to PLAG1 rearrangement. Based on these seemingly contradictory findings, we raise the question on what grounds neoplastic diseases are best classified, namely, their genotypic or phenotypic characteristics. 


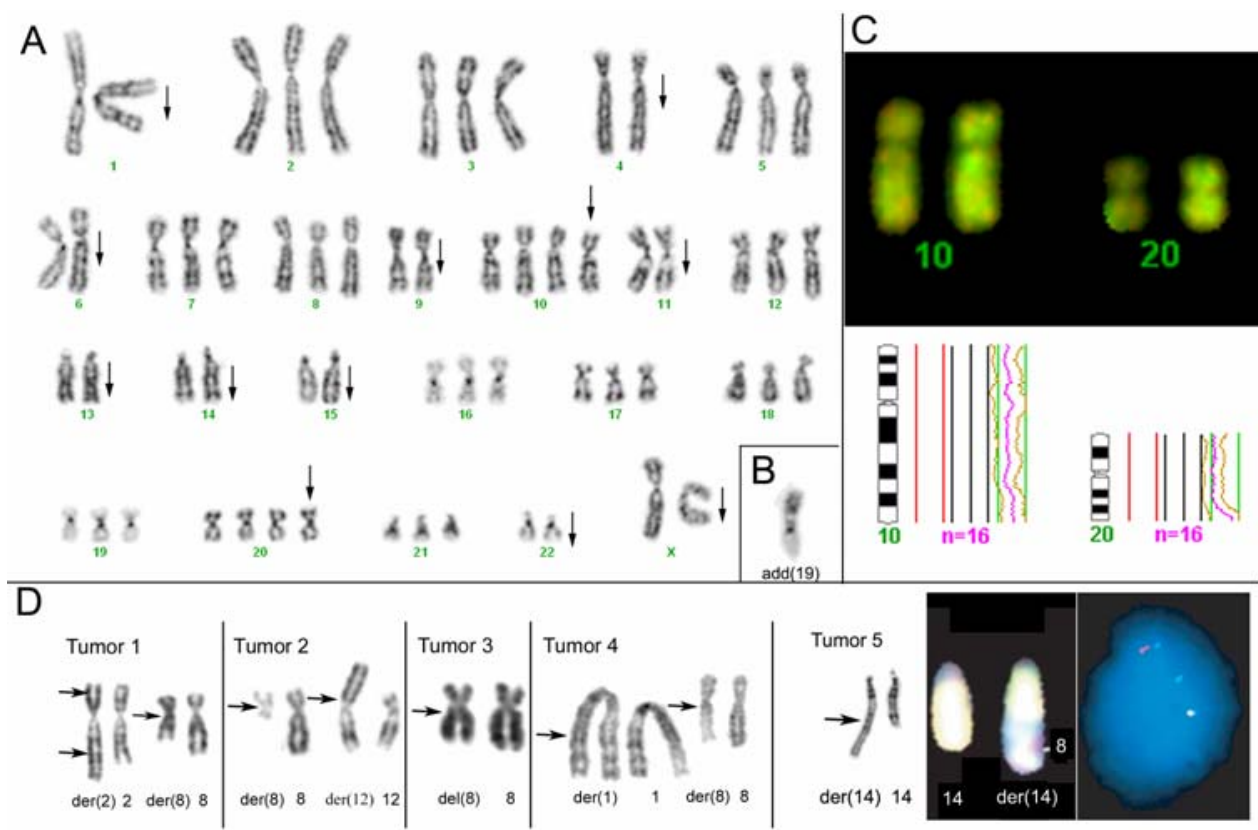

FIGURE 1. (A) Karyogram showing only numerical changes from the first-ever karyotyped reninoma. Arrows indicate missing or extra chromosome copies relative to the triploid level. (B) The only structurally aberrant chromosome found in the same reninoma, add(19)(p13). (C) When comparative genomic hybridization was performed, gains of chromosomes 10 and 20 were the only scorable findings in this reninoma. Shown here are the reverse in situ hybridization pictures as well as the gain/loss profile for these two chromosomes. (D) The aberrant chromosomes in the five lipomatous tumors studied are shown alongside their normal chromosome homologs; again, arrows indicate break points. For tumor 5, we show the G-banded chromosomes 14 with added material of unknown origin to one of them, a multicolor fluorescence in situ hybridization (FISH) picture of the same chromosomes revealing that the added material is of chromosome 8 origin, and an interphase FISH picture of a nucleus from the same tumor with three signals for the PLAG1-containing probe (green) and two signals for the more proximal 8q probe (red), suggesting PLAG1-involvement in the break point.

\section{REFERENCES}

1. Brandal P, Micci F, Bjerkehagen B, Eknæs M, Larramendy M, Lothe RA, Knuutila S, Heim S. Molecular cytogenetic characterization of desmoid tumors. Cancer Genet Cytogenet. 2003;146(1):1-7.

2. Brandal P, Bjerkehagen B, Heim S. Molecular cytogenetic characterization of tenosynovial giant cell tumors. Neoplasia. 2004;6(5):578-83.

3. Brandal P, Bjerkehagen B, Danielsen H, Heim S. Chromosome 7 abnormalities are common in chordomas. Cancer Genet Cytogenet. 2005;160(1):15-21.

4. Brandal P, Busund LT, Heim S. Chromosome abnormalities in juxtaglomerular cell tumors. Cancer. 2005;104(3):504-10. 
5. Brandal P, Lie AK, Bassarova A, Svindland A, Risberg B, Danielsen H, Heim S. Genomic aberrations in mucinous tubular and spindle cell renal cell carcinomas. Mod Pathol. 2006;19(2):186-94.

6. Brandal P, Bjerkehagen B, Heim S. Rearrangement of chromosomal region 8q11-13 in lipomatous tumors: Correlation with lipoblastoma morphology. J Pathol. 2006;208(3):388-94.

\section{Comment by Professor Janusz Limon, Chair of Department of Biology and Genetics, Collegium Biomedicum, Medical University of Gdańsk, 80-211 Gdańsk, 1 Dębinki St., Poland}

There are several reasons why the cytogenetics of mesenchymal tumors is interesting. First, pioneer cytogenetic studies have unraveled relatively simple and sometimes specific chromosome changes in some mesenchymal entities. Second, because mesenchymal tumors are often difficult to diagnose, we need better tools to distinguish them from one another. Third, molecular studies of genes involved in specific chromosomal translocations in some of these tumors are diagnostically important and reveal some of the pathogenetic mechanisms involved in tumor development.

The main source of chromosomes is from dividing cells in primary tissue cultures established from collagenase disaggregated tumor tissue. However, the isolation of chromosomes from solid tumors is difficult for several reasons, namely, lack of fresh tissue specimens, microbiological contamination, lack of cancer cells in the tissue specimens, and others. Complementary methods like fluorescence in situ hybridization (FISH) and comparative genomic hybridization (CGH) have been introduced, and the bulk of the experiments in this thesis were performed on archival, fixed material. Such material is very difficult to work with and there is a risk of obtaining false results, especially in molecular studies. For the above reasons, the clearly formulated aims of Petter Brandal's thesis are matters of current importance in human tumor cytogenetics; first, to search for cytogenetic aberrations in tumor entities that have not been well studied cytogenetically before, and second, to evaluate the usefulness of molecular cytogenetic techniques [in particular CGH and interphase-FISH (IP-FISH)] for cytogenetic characterization of selected tumor types. Both approaches provide a chance to elucidate tumorigenic mechanisms in clearly neoplastic tumors and, very importantly, investigate the pathogenesis of disease entities of unknown nature.

The material of the thesis was carefully selected and several rare tumor types were analyzed. Furthermore, the number and quality of used techniques is impressive; from cell culturing and simple karyotyping to CGH, IP-FISH, multiplex-FISH, microsatellite instability analysis, and DNA ploidy analysis. I would like to underline that some of the results are original and bring new data into the 
field of tumor cytogenetics. The author's most important findings are as follows:

1. Molecular cytogenetic characterization of desmoid tumors showing lack of chromosome changes in the majority of the tumors

2. The description of chromosome aberrations that are probably pathogenetically important in tenosynovial giant cell tumors (trisomy 7 in 56\% of tumors)

3. Cytogenetic characterization and additional molecular information about chordomas

4. The first description of chromosomal changes in the rare kidney tumor entity juxtaglomerular cell tumor, and genomic information about a relatively new diagnostic entity, mucinous tubular and spindle cell carcinoma of the kidney

5. Cytogenetic characterization of lipomatous tumors with 8q11-13 rearrangements

6. The attempt to elaborate on methodological considerations in the molecular cytogenetic studies of human solid tumors

I would like to stress the originality of the work, the unique material, and the well-grounded methodology, and I would like to underline that Brandal's thesis provides new pathogenetic information and thus contributes to the knowledge of the cytogenetics of rare human solid tumors. 